\title{
Numerical Simulation of the Flow around Marine
}

\section{Propeller Series}

\author{
Djahida Boucetta and Omar Imine \\ Department of Marine Engineering, Faculty of Mechanical Engineering, University of Sciences and Technology of Oran, B.P 1505 \\ USTO, Oran, Algeria
}

\begin{abstract}
The objective of marine propeller design optimization study is to obtain a propeller with minimum power absorption, maximum efficiency and good materials resistance. In this study, results of numerical simulation carried out on the flow around a conventional marine propeller are presented. The investigation focused on the aspects related to the influence of skew magnitude, thickness and blade number on the propeller performances. First, open water performances of a conventional propeller model DTMB 4148 was estimated using RANS (Reynolds Averaged Navier-Stokes) method. The flow around rotating propeller model was analyzed in the steady state using RANS approach of the commercial CFD (computational fluid dynamics) code fluent. The results provide good agreement with literature data. Numerical results show that the number of blades has an influence on the open water performances of marine propellers. It's noticed that the best propeller has four or five blades from only the hydrodynamic aspect. The thickness blade effect has been studied for the same propeller model and compared to the blade with three different thickness values. Results of the calculation show that the blade thickness increases moderately the propeller efficiency. Finally, numerical simulation is performed to study the magnitude skew effect on the propeller blade performance, so three different models were generated. The results of the simulation show that the skew distribution has a positive effect on the open water performances of the marine propellers.
\end{abstract}

Key words: Marine propeller, CFD simulation, blade number, blade thickness, skew magnitude, open water performances.

\section{Nomenclature}

$\begin{array}{ll}D: & \text { propeller diameter } \\ C_{r}: & \text { propeller chord length } \\ P: & \text { propeller pitch } \\ Z: & \text { blade number } \\ t: & \text { propeller blade thickness } \\ A_{E}: & \text { blade expended area } \\ n: & \text { number of propeller revolutions } \\ V_{a}: & \text { propeller advance velocity } \\ J: & \text { advance coefficient } \\ T: & \text { thrust } \\ Q: & \text { torque } \\ K_{T}: & \text { thrust coefficient } \\ K_{Q}: & \text { torque coefficient } \\ \eta_{0}: & \text { propeller efficiency in open water } \\ C_{P}: & \text { pressure coefficient } \\ \rho: & \text { density of water }\end{array}$

\section{Introduction}

In order to get the optimum propeller design

Corresponding author: Djahida Boucetta, Ph.D., research fields: marine propellers, fluid dynamic, cavitation. geometries with highest efficiency, RANS (Reynolds Averaged Navier-Stokes) method became a practical useful tool which replaces the methods based on the potential theory. Many researchers have contributed to the improvement of open water performances of marine propellers using RANS CFD (computational fluid dynamics) code such as Mishima in Ref. [1]. They developed a numerical method to determine the blade geometry with the best efficiency. Their method was also extended to include the skew distribution. Chang [2] applied a finite volume CFD method in conjunction with the standard $\mathrm{k}-\varepsilon$ turbulence model to calculate the flow pattern and performance parameters of a DTNSRDC P4119 marine propeller in a uniform flow. Watanabe et al. [3] applied unstructured grid technique to the flow around the Sieun-maru propeller. The agreement with experiment was good both for steady and unsteady conditions. The RANS methods were later applied [4] to the design of ducted 
propellers. Further, analysis and design techniques for ducted propellers can be found in the works of kinnas [5]. The optimization of marine propellers shape was presented [6-9]. A MOPSO (Multi-Objective Particle Swarm Optimization) was employed; the two objectives identified are maximizing efficiency and minimizing cavitation. They also investigate the impacts of shape, number of blades and operating conditions on both objectives. A practical design method to obtain optimum cavitating ship propeller by combining vortex lattice lifting line method and lifting surface method for DTMB 4119 and DTMB 4381 propellers was applied [7], a very good level of satisfaction was obtained. Noise prediction method for cavitating marine propellers DTMB 4148 was presented in [8]. In the same work two different propellers (DTMB 4119 and Seiun-maru highly skewed propeller) are analyzed by lifting surface and CFD methods. It is concluded that this last approach provides a helpful tool for the propeller pre-design.

The present paper deals with a complete computational solution for the flow around conventional marine propeller (DTMB 4148) using fluent software. Open water performances were estimated and compared with literature data. To get the optimum propeller geometry many calculations were developed focused on changing the blade shape. In this paper, the variation of the number of blades, skew magnitude and blade thickness is taken into account.

\section{Characteristics of Marine Propellers}

The efficiency of the propulsion system is strongly dependent on thrust force, torque of propeller and its efficiency. Therefore, to evaluate the marine propeller hydrodynamic performances is to graph the coefficients $\mathrm{K}_{\mathrm{T}}, \mathrm{K}_{\mathrm{Q}}, \eta_{0}$ versus the advance coefficient $\mathrm{J}$ [10-12].

$\mathrm{J}$ is the advance ratio of the propeller and calculated as the following:

$$
J=\frac{V_{a}}{n \times D}
$$

The open water efficiency $\eta_{0}$ given in Eq. (2) is established for a propeller working in a homogeneous flow without any ship hull.

$$
\eta_{0}=\frac{K_{T}}{K_{Q}} \cdot \frac{J}{2 \pi}
$$

where both of thrust and torque coefficient are evaluated from the diameter $\mathrm{D}$ and the revolutions number $\mathrm{n}$ of the propeller.

$$
\begin{gathered}
K_{T}=\frac{T}{\rho \cdot n^{2} \cdot D^{4}} \\
K_{Q}=\frac{Q}{\rho \cdot n^{2} \cdot D^{5}}
\end{gathered}
$$

\section{Geometric Modeling}

The present paper deals with a complete computational solution for the flow around a conventional marine propeller model DTMB 4148 by using fluent code. Table 1 shows the data and geometry of the model propeller used in this study. The non-dimensional blade geometry data of the propeller is presented in Table 2 [8]. These data were converted into coordinate point to generate the surface model of propeller by using a FORTRAN Program. A solid model of the propeller was created in Gambit as shown in Fig. 1.

\subsection{Grid Generation}

A solid model of the propeller was created in Gambit as a first step of grid generation. Due to the complexity of marine propeller geometry, unstructured tetrahedral mesh has been adopted. Taking into account that the flow is circumferentially periodic, only one angular sector of 120 degrees containing

Table 1 Propeller data of DTMB 4148.

\begin{tabular}{ll}
\hline Blade number & 3 \\
\hline Propeller diameter $(\mathrm{m})$ & 0.3048 \\
Propeller rate of rotation per second (1/s) & 17.17 \\
Advance coefficient $J_{s}$ & 0.954 \\
Skew (degree) & 0 \\
Rake (degree) & 0 \\
\hline
\end{tabular}


Table 2 Blade characteristics of the DTMB 4148 model propeller.

\begin{tabular}{lllll}
\hline $\mathrm{r} / \mathrm{R}$ & $\begin{array}{l}\text { Chord } \\
\text { distribution } \\
\left(C_{r} / \mathrm{D}\right)\end{array}$ & $\begin{array}{l}\text { Pitch } \\
\text { distribution } \\
(\mathrm{P} / \mathrm{D})\end{array}$ & $\begin{array}{l}\text { Thickness } \\
\text { distribution } \\
(\mathrm{t} / \mathrm{D})\end{array}$ & $\begin{array}{l}\text { Camber } \\
\text { distribution } \\
(\mathrm{f} / \mathrm{D})\end{array}$ \\
\hline 0.2 & 0.16 & 0.9921 & 0.0329 & 0.0174 \\
0.3 & 0.1818 & 0.9967 & 0.0282 & 0.0195 \\
0.4 & 0.2024 & 0.9987 & 0.0239 & 0.0192 \\
0.5 & 0.2196 & 0.9975 & 0.0198 & 0.0175 \\
0.6 & 0.2305 & 0.9944 & 0.0160 & 0.0158 \\
0.7 & 0.2311 & 0.9907 & 0.0125 & 0.0143 \\
0.8 & 0.2173 & 0.9850 & 0.0091 & 0.0133 \\
0.9 & 0.1806 & 0.9788 & 0.0060 & 0.0125 \\
0.95 & 0.1387 & 0.9740 & 0.0045 & 0.0115 \\
1 & 0.0010 & 0.9680 & 0 & 0 \\
\hline
\end{tabular}

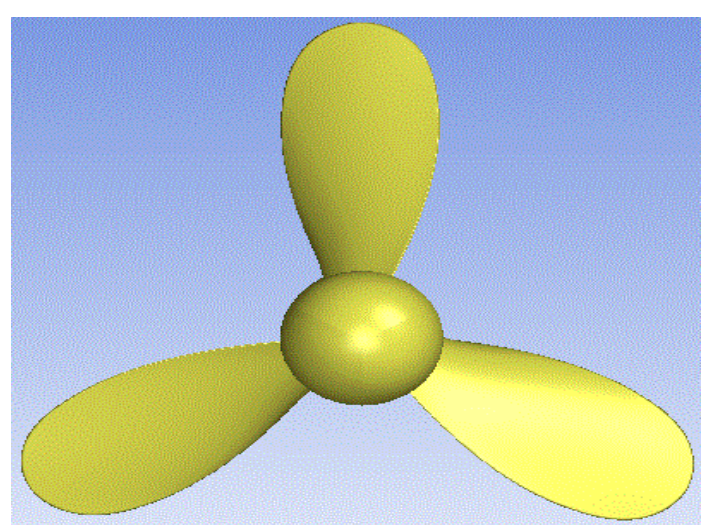

Fig. 1 Perspective view of DTMB 4148 propeller blades.

one blade was modeled. The solution domain represents $1 / 3$ of a cylinder with its inlet located at $1.5 \mathrm{D}$ upstream, and its outlet located at $3.5 \mathrm{D}$ downstream of propeller plane [13]. In radial direction, the domain was considered up to a distance of 1.4D from the axis of the hub. This peripheral plane is called far-field boundary. Fig. 2 shows the computational domain and boundary condition used for the simulation. Different zones with refined meshes have been introduced around the cross section of the blade at the hub intersection and near the blade tip. A mesh refinement zone is defined near the propeller surface in order to capture the high gradients in the flow. The mesh was generated in such a way that cell sizes near the blade wall were small and increased progressively towards outer boundary. To resolve the turbulent boundary layer on the blade surfaces TGrid has been used [14]. Ten layers of prismatic cells were growing from the blade and hub surface where the first cell height was $0.00001 \mathrm{D}$ and the growth ratio of the layer was 1.1. Fig. 3 shows the grid over the entire blade and hub propeller. Finally, all of the calculation zone and domain are meshed by tetrahedral meshes as shown in Fig. 4. Three meshes were generated and tested for predicting the open water performances of the propeller model as resume Table 3 .

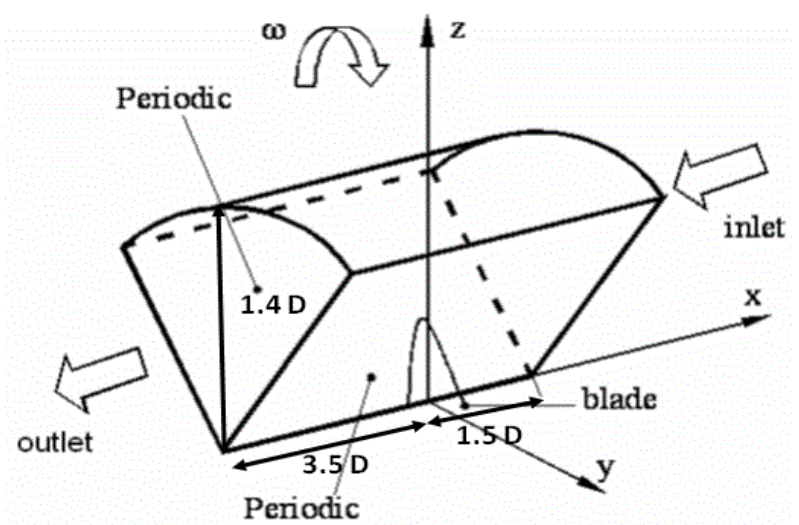

Fig. 2 Computational domain and boundary conditions.

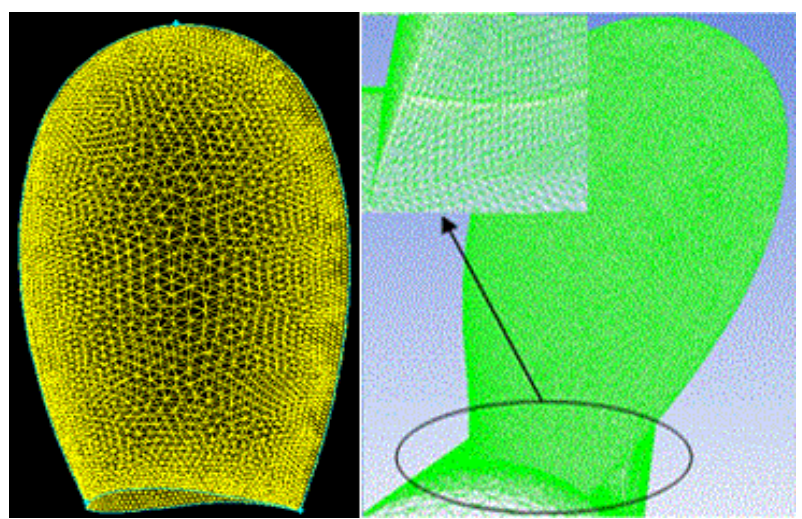

Fig. 3 Mesh on surface blade and boundary layer.

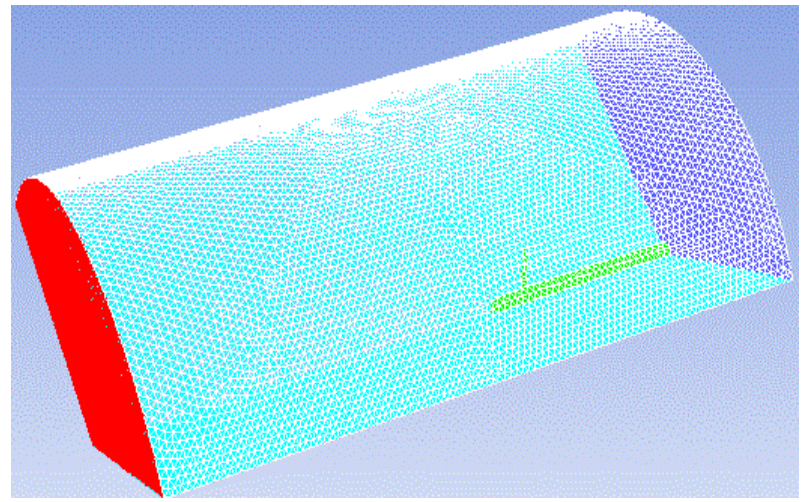

Fig. 4 Grid over the computational domain. 
Table 3 Blade characteristics of the DTMB 4148 model propeller.

\begin{tabular}{llll}
\hline & Element type & N nodes & Mesh type \\
\hline Mesh 01 & Tetrahedral & 80,000 & coarse \\
Mesh 02 & Tetrahedral & 160,000 & medium \\
Mesh 03 & Tetrahedral & 300,000 & fine \\
\hline
\end{tabular}

\section{Solution and Solver Settings}

In this study, results of a numerical simulation carried out on the flow around a conventional marine propeller are presented. The investigation focused on the aspect related to the influence of skew magnitude, thickness and blade number on the propeller performances. First, open water performances of a conventional propeller model DTMB 4148 was estimated in the steady state using the RANS method. Three meshes were generated and tested in order to define the optimum mesh representing the flow around rotating propeller. A MRF (Moving Reference Frame) option was employed to solve the governing equations for the rotating propeller [15-16]. The SST (Shear Stress Transport) K- $\omega$ model is chosen for turbulence closure. The simple algorithm has been adopted for the velocity-pressure coupling and the schemes were all in the second order. The inlet velocity is changing in such a way to reach the advance coefficient between $\mathrm{J}=0.3$ and $\mathrm{J}=0.9$ and the rotational speed of propeller was kept constant (n $=17.17 \mathrm{r} / \mathrm{s}$ ). Pressure outlet boundary conditions have been adopted at the exit. The wall forming the propeller blade and hub were assigned at the non-slip boundary condition and the far boundary (far field) were taken as inviscid wall.

\section{Results and Discussion}

To validate the numerical method, open water performances of the flow around rotating propeller model DTMB 4148 was analyzed in the steady state using the RANS method. The quality of the mesh is considered a key factor in the success of a numerical simulation. Therefore, the propeller model was meshed for three sets of grid; coarse, medium and fine to have more accurate results. The curves of $\mathrm{K}_{\mathrm{T}}, \mathrm{K}_{\mathrm{Q}}$ and $\eta_{0}$ against advance coefficient $J$ were presented in Fig. 5, Fig. 6 and Fig. 7 respectively. Globally, a same trend for the all coefficients is observed. It is important to note that the refined mesh gives more reliable results compared to the two others. This type of mesh has been chosen for all next simulations.

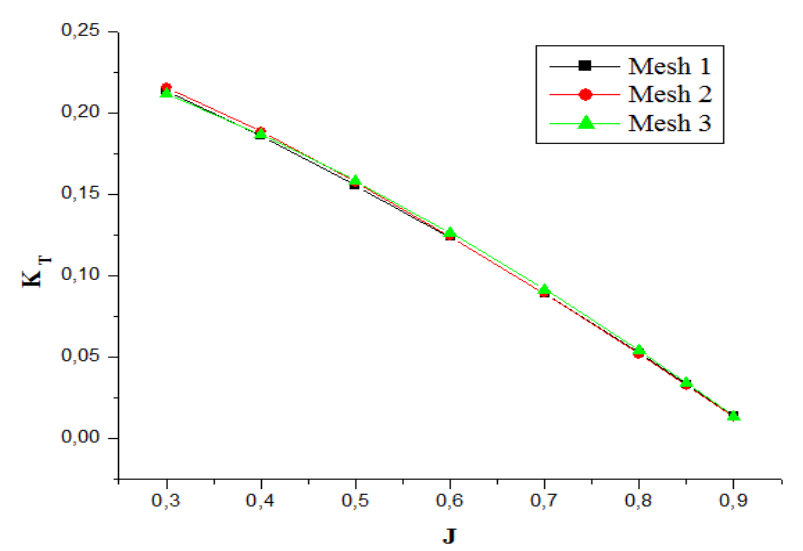

Fig. 5 Comparison of $K_{T}$ values with different mesh size.

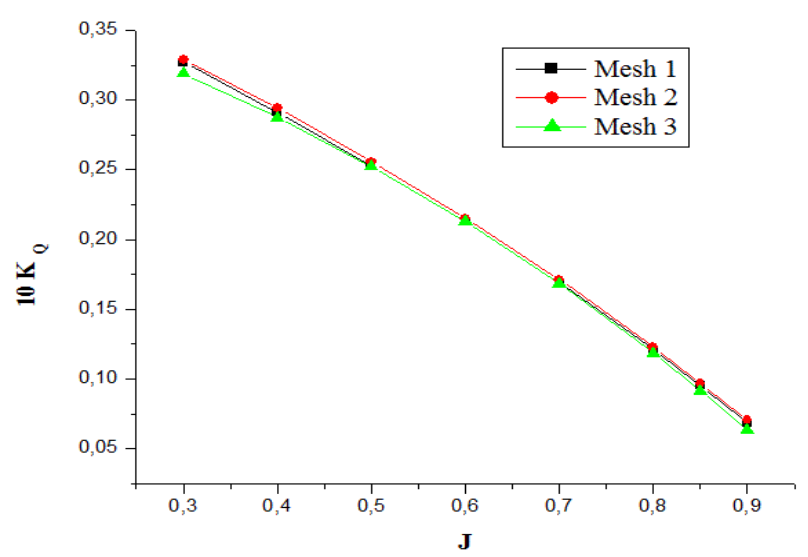

Fig. 6 Comparison of $K_{Q}$ values with different mesh size.

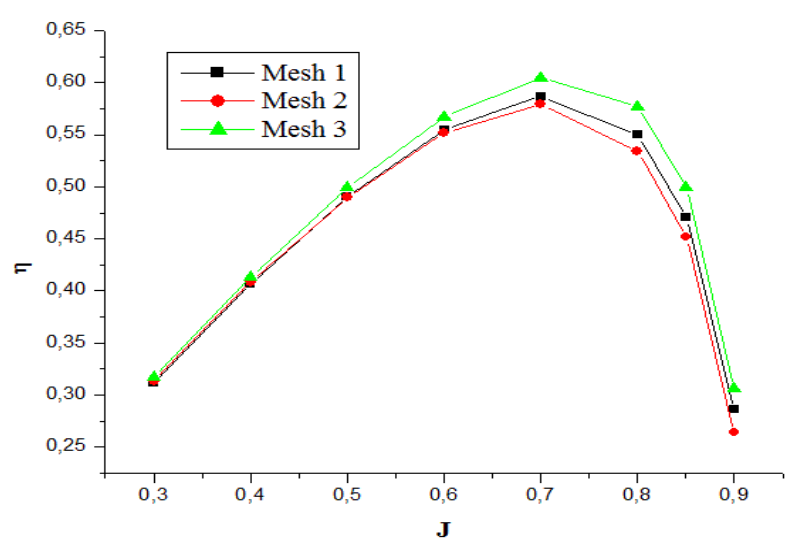

Fig. 7 Comparison of $\eta_{0}$ values with different mesh size. 
All simulations done in this work have been performed with wall treatment providing wall $\mathrm{y}^{+}<3$ as shown in Fig. 8.

The numerical simulation permits to get the field of pressure on the propeller blade faces. Fig. 9 shows the contour of pressure coefficient for $\mathrm{J}=0.7$. The exam of these contours provide that the peak value $\left(-c p_{\min }\right)$ appears near the leading edge of the upper pressure side where the minimal value is encountered on the down pressure face.

Numerical simulation is performed to study the blade number effect on the open water performances. The geometry of the DTMB 4148 propeller model with three blades is modified by adding one and two blades but the surface AE is maintained constant, Eq. 5 . For this, the geometry has been modified according to the following chord law, Eq. 6 [17-18]. The Fig. 10 illustrates the perspective views of the propeller with different blade number. Numerical results provide that the number of blades has an influence on the open water performances of marine propellers as shown in

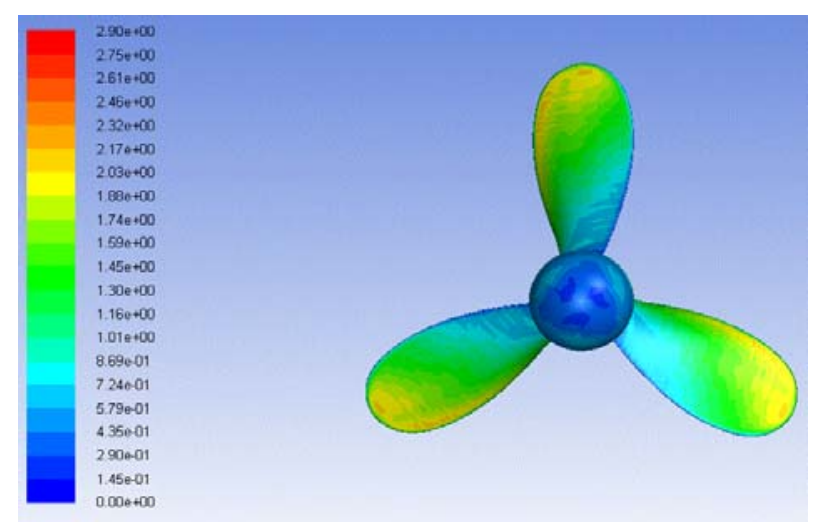

Fig. 8 Contours of wall $\mathbf{y}^{+}$.

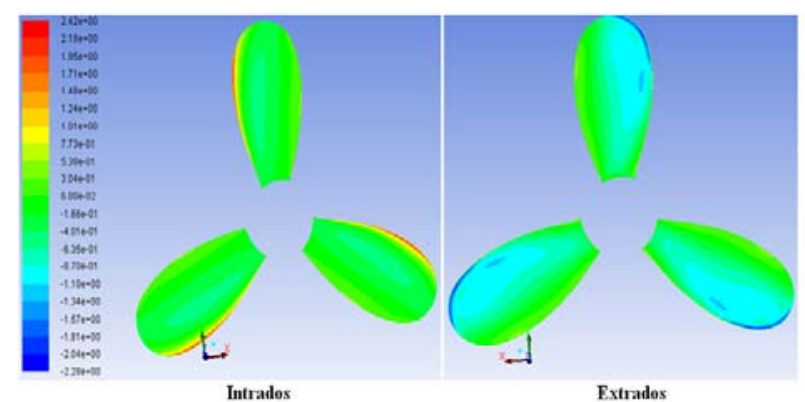

Fig. 9 Contours of pressure coefficient on the blade propeller faces.
Fig. 11. From the point-view of the hydrodynamic, it appears that the best propeller has 4 blades.

$$
\begin{gathered}
A_{E}=Z \int_{R_{h}}^{R_{P}} C_{r} d r=Z \int_{R_{h}}^{R_{p}} C_{r}{ }^{*} d r \\
C_{r}{ }^{*}=C_{r} \frac{Z}{Z^{*}}
\end{gathered}
$$

The thickness blade effect has been studied by considering three configurations which were generated by multiplying the thickness of the basic model by a coefficient. Fig. 12 shows the curve of $\eta_{0}$ as function of $\mathrm{J}$ with the corresponding measured values. It was also noticed that results of the calculation show globally that the increase of blade thickness increases substantially the propeller efficiency.

The open water characteristics were determined by calculation for three propellers with different skew distributions: the DTMB 4148 propeller with Skew angle 0 deg, positive skew angle and negative skew angle as shown in Fig. 13. The results of the computation

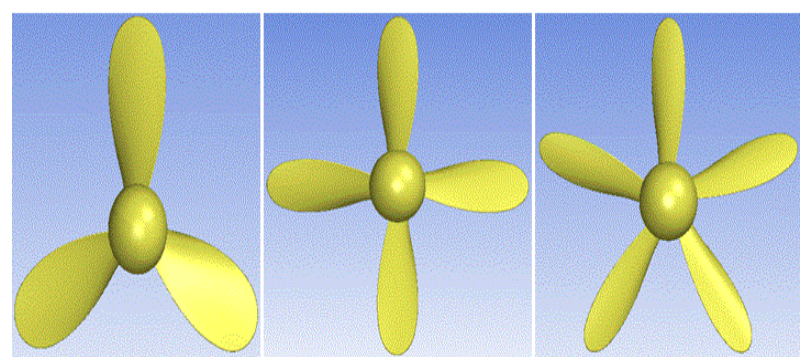

Fig. 10 Perspective view of DTMB 4148 propeller with different blade number.

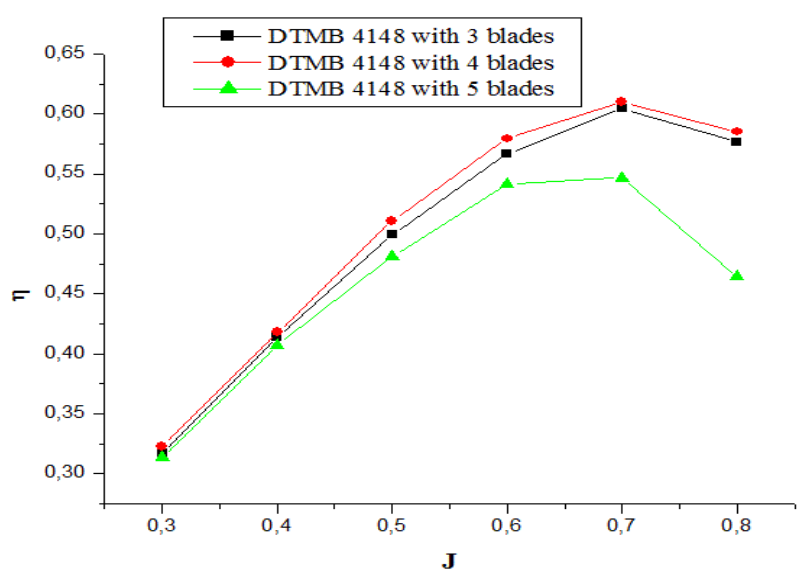

Fig. 11 Blade number effect on the propeller efficiency. 


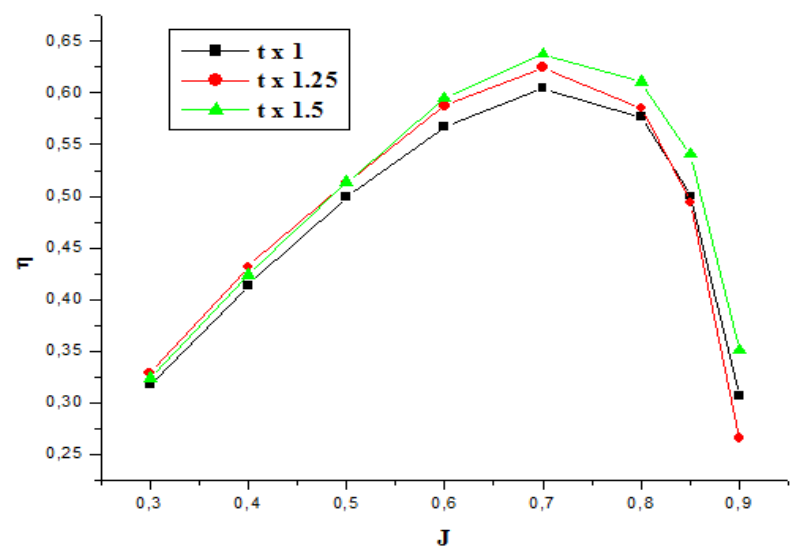

Fig. 12 Thickness blade effect on the propeller efficiency.

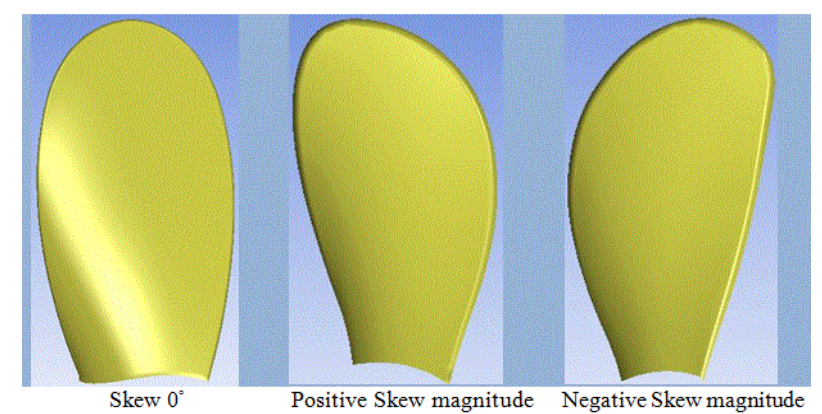

Fig. 13 General view of propeller blades with different skew angles.

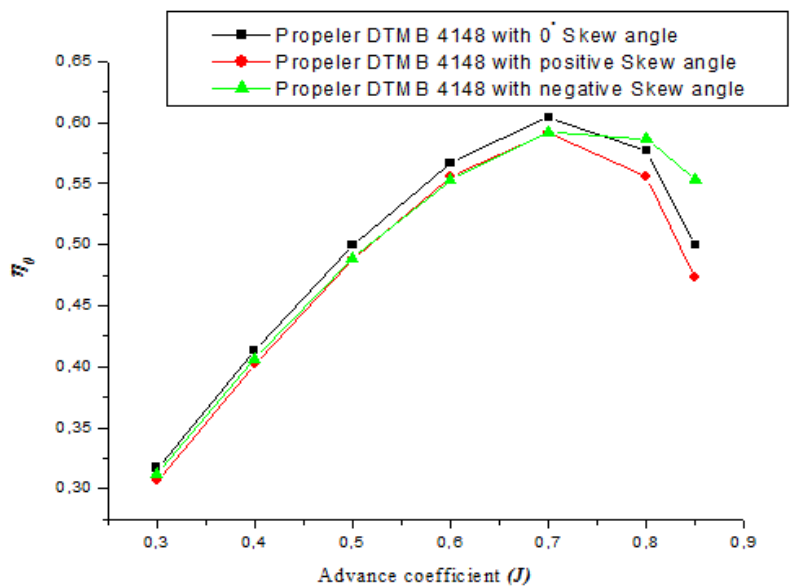

Fig. 14 Comparison of $\eta_{0}$ values with different skew angles.

show that the skew distribution has a positive effect on the open water performances of tested propellers as is illustrated in Fig. 14.

\section{Conclusions}

In this study, results of a numerical simulation carried out on the flow around a conventional marine propeller are presented in order to validate the proposed approach and simulate the effect of some geometric aspects. Open water performances of a conventional propeller model DTMB 4148 was estimated in the steady state using the RANS method. The DTMB 4148 propeller, with three blades, has been chosen for the validation as reference model. Therefore, three grids with different mesh quality have been tested: coarse, medium and refined in order to define the minimal doorstep for the grid promising a good stability of the results. Although this condition has been assured from the medium grid, the refined mesh has nevertheless been adopted for the rest of the survey. Moreover, a particular attention has been made to study the effects of number and thickness of blades as well as the angle of skew on the hydrodynamic performances. The results show that the number of blades has a positive influence on the open water characteristics of the marine propeller and the propeller with four blades gives the best efficiency. Besides, this investigation shows that the increase of blade thickness generates a raise in propeller efficiency. Finally, the adoption of a skew angle on the blade improves the hydrodynamic performances of marine propeller.

\section{References}

[1] Mishima, S., and Kinnas, S. A. 1997. "Application of Numerical Optimization Technique to the Design of Cavitating Propellers in Non-uniform Flow.” Journal of Ship Research 41: 93-107.

[2] Chang, B. 1998. "Application of CFD to P4119 Propeller." Presented at the 22nd ITTC Propeller RANS/Panel Method Workshop, France.

[3] Watanabe, T., Kawamura, T., Takekoshi, Y., Maeda, M., and Rhee, S. H. 2003. "Simulation of Steady and Unsteady Cavitation on a Marine Propeller Using a RANS CFD Code." Presented at the Fifth International Symposium on Cavitation (CAV2003), Japan.

[4] Kerwin, J. E. 2003. "The Preliminary Design of Advanced Propulsors." In Proceedings of Propellers/Shafting 2003 Symposium, SNAME: 16-7.

[5] Kinnas, S. A., Lee, H. S., Gu, H., and Deng, Y. 2005. "Prediction of Performance and Design via Optimization of Ducted Propellers Subject to Non-axisymmetric Inflows." Trans. SNAME 113: 99-121.

[6] Mirjalili, S., Lewis, A., and Mirjalili, S. M. 2015. 
"Multi-objective Optimisation of Marine Propellers." In Proceedings of ICCS 2015 International Conference on Computational Science, published by Procedia Computer Science 51: 2247-56.

[7] Bal, S. 2011. "A Method for Optimum Cavitating Ship Propellers." TUBITAK, Turkish J. Eng. Env. Sci. 35: 139-58.

[8] Ekinci, S., Çelik, F., and Guner, M. 2010. "A Practical Noise Prediction Method for Cavitating Marine Propellers." BRODOGRADNJA 61 (4): 359-66.

[9] Krasilnikov, V., Sun, J., and Halse, K. 2009. “CFD Investigation in Scale Effect on Propellers with Different Magnitude of Skew in Turbulent Flow." In Proceedings of the First International Symposium on Marine Propulsors.

[10] Ekinci, S. 2011. "A Practical Approach for Design of Marine Propellers with Systematic Propeller Series." BRODOGRDNJA 62 (2): 123-9.

[11] Gaafary, M. M., El-kilani, H. S., and Moustafa, M. M. 2010. "Optimum Design of B-series Marine Propellers." Alexandria Engineering Journal 50: 13-8.

[12] Nakisa, M., Abbasi, M. J., and Amini, A. M. 2010.
"Assessment of Marine Propeller Hydrodynamic Performance in Open Water via CFD." In Proceedings of MARTEC 2010.

[13] Kawamura, T., Takekoshi, Y. 2006. "Simulation of Unsteady Cavitating Flow around Marine Propeller Using a CFD Code."

[14] Amoraritei, M. 2005. "Application of CFD in Analysis of Conventional Propeller and Azimuth Thruster." In Proceedings of Workshop on Vortex Dominated Flowsc -Achievements and Open Problems.

[15] Sheng, H., Xiang-yuan, Z. 2007. "CFD Simulation of Propeller and Rudder Performance When Using Additional Thrust Fins." Journal of Marine Science and Application 6 (4): 27-31.

[16] Kim, M., Chun, H., and Kang, D. 2004. "Design and Experimental Study on a New Concept of Preswirl Stator as an Efficient Energy Saving Device for Slow Speed Full Body Ship." Pusan National University, Korea.

[17] Carlton, J. 2007. Marine Propeller and Propulsion. 2nd Edition. Great Britain, Bodmin Cornwall: MPG Books Ltd.

[18] Presles, D. 2005. Architecture Navale. Paris: Edition de la Villette. 